\title{
Emission of a low-power laser-induced vacuum discharge plasma in the EUV and SXR spectral ranges
}

\author{
Alexander Rupasov ${ }^{1}$, Igor Romanov ${ }^{1}$, Andrey Kologrivov ${ }^{1}$, and Viktor Paperny ${ }^{2}$ \\ ${ }^{1}$ Lebedev Physical Institute, Russian Academy of Sciences, Moscow, Russia \\ ${ }^{2}$ Irkutsk State University, Irkutsk, Russia
}

\begin{abstract}
X-ray spectral characteristics of a vacuum discharge plasma with the storage energy lower than $30 \mathrm{~J}$ initiated on an $\mathrm{Al}$ or a Fe cathode by a $10^{12} \mathrm{~W} / \mathrm{cm}^{2}$ neodymium laser were studied in the $30-300 \AA$ wavelength range. It is shown that both the spectral composition and intensity of radiation of a micropinch plasma produced in the cathode jet of the discharge are determined by parameters of the discharge and laser pulse. These parameters were optimized to achieve a regime in which a considerable part of radiation energy was concentrated in the long-wavelength band of the quasi-continuum (230 - $270 \AA$ and 160 $200 \AA$ for $\mathrm{Al}$ and $\mathrm{Fe}$, respectively), which makes this discharge a source of narrowband X-ray radiation.
\end{abstract}

It is known that local regions of a hot micron-scale plasma produced in a high-current vacuum discharge plasma due pinching (micropinches) are sources of intense electromagnetic radiation in a broad spectral range. The emission spectra of micropinches in the hard $\mathrm{X}$-ray range at wavelengths $\lambda<2 \AA$ were studied in many works (see, for example, [1-3]). Soft X-ray (SXR) sources in the wavelength range above $20 \AA$ attract considerable recent interest due to their possible scientific and technological applications including nanolithography, investigations of the inner structure of biological objects, astrophysical studies, etc. [4-7]. At present, as sources of such pulsed radiation, laser plasmas are mainly used. An important parameter for applications of radiation sources is the SXR conversion efficiency from a storage electric energy. From this point of view, it is interesting to use micropinch radiation sources. It was shown earlier that in vacuum discharge plasma with the storage energy about $10 \mathrm{~J}$ initiated on a cathode by a low-power laser pulse, micropinches emitting SXRs in the wavelength region $>40 \AA$ are formed. The plasma pinching process stability and micropinch parameters were controlled by the laser pulse parameters $[8,9]$. These results suggest the possibility of making a local source of intense SXR with a comparatively low energy deposition based on the laserinduced vacuum discharge.

The use of such a discharge as a SXR source requires the knowledge of the spectral composition of the radiation depending on the parameters of the discharge, laser pulse and cathode material. In turn, the maximum temperature of the micropinch plasma can be determined from shape of the emission spectrum of a metal plasma of some elements, which is important both for understanding the physics of processes involved and for improving parameters of the SXR source based on this discharge. However, the long-wavelength region of the SXR spectrum remains poorly studied, which is explained by the complexity of required measurements and their interpretation. Note that the mega-ampere Z-pinch plasma was studied in $[10,11]$ where only a few spectra were presented for fixed discharge parameters.

In this paper, we present the measurements of the emission spectrum of a micropinch plasma produced in the cathode jet of a low-power discharge in a broad spectral range from 30 to $300 \AA$ for different laser pulse energies, different discharge voltages (currents), and different cathode materials.

Investigations were performed on a laboratory stand for studying X-ray and ion emission from discharge plasmas. The setup included a vacuum diode, a Q-switched $1.06 \mu \mathrm{m}$ laser emitting $\leq 500 \mathrm{~mJ}, 6 \mathrm{~ns}$ pulses and also X-ray and corpuscular diagnostics. The scheme of experiments is shown in Fig. 1. The current in the discharge circuit was maintained with a storage $0.22 \mu \mathrm{F}$ capacitor. The current was measured with a Rogowski coil placed in the cathode circuit of the diode. The semiperiod of current oscillations in the shorting regime was $0.4 \mu$ s. Experiments were performed for discharge current amplitudes no more than $28 \mathrm{kA}$ and storage voltages up to $-16 \mathrm{kV}$. The maximum current rise rate was $5 \cdot 10^{11} \mathrm{~A} / \mathrm{s}$. The discharge was initiated on the cathode surface by a laser beam focused to a fine $0.4 \cdot 10^{-4}$ $\mathrm{cm}^{2}$ spot. The laser pulse power density was varied between $10^{10}-10^{12} \mathrm{~W} / \mathrm{cm}^{2}$. The angle of incidence of the laser beam on the cathode was $45^{\circ}$. We used in experiments a ground steel cathode with a hole $6 \mathrm{~mm}$ in diameter in the middle (for ion emission experiments). 
The interelectrode gap was $7 \mathrm{~mm}$. The diode was placed in a chamber with oil-free pumping down to a residual pressure of $10^{-4} \mathrm{~Pa}$. After each or several discharges, the electrode unit was displaced and the laser beam fell on a new spot on the cathode.

The discharge plasma radiation was studied in the integrated regime with a GIS-S grazing incidence spectrograph mounted perpendicular to the interelectrode gap at a distance of $65 \mathrm{~cm}$ from it. The entrance slit of the spectrograph was oriented along the discharge axis.

A concave $600 \mathrm{1} / \mathrm{mm}$ diffraction grating of the spectrograph with period $d=1.667 \mu \mathrm{m}$, the radius of curvature $1 \mathrm{~m}$, and the grazing angle $4^{0}$ has a W/Re coating. The non-Rowland configuration is used in which the spectrum is recorded in a plane perpendicular to diffracted beams. Thus, the accurate focusing is performed only for one wavelength corresponding to the point of intersection of the recording plane with the Rowland circle (accurate focusing point). For our spectrograph, the accurate focusing point corresponds to the wavelength $\lambda=140 \AA$. Because of a small angular aperture of the spectrograph, the beam defocusing in the non-Rowland configuration is small and therefore the spectrum can be recorded in a broad enough spectral range. The spectral resolution $\delta \lambda$ for this configuration and the $20 \mu \mathrm{m}$ exit slit width of the spectrograph is $0.66 \AA$ in the accurate focusing region (where $\lambda / \delta \lambda \approx 200$ ) and $\sim 1 \AA$ at the range boundaries. Note that the recording range is restricted in the short-wavelength region by the drastic decrease in the reflection coefficient of the grating at $\lambda<30 \AA$. Measurements of the $48.41 \AA$ Ly-alpha line intensity for the B V ion in the first and second diffraction orders performed during spectrograph calibration showed that the second-order line intensity was $\sim 15$ times weaker than that in the first diffraction order [12]. Such a strong suppression of the second order allows us to neglect it in the processing of spectrograms, taking into account only the first order in the $30-90 \AA$ region. For wavelengths above $90 \AA$, it is necessary to take into account the third order and above $120 \AA$ - also the fourth order (radiation in the third and fourth diffraction orders is approximately two times attenuated compared to the first order). Thus, the wavelength range from 30 to $90 \AA$ is "pure" from the overlap of diffraction orders, whereas at longer wavelengths the spectrum recovery procedure should be applied, which was not performed here.

Spectra were recorded with a Toshiba TCD 1304A linear CCD array coated with a P-46 luminophore converting SXRs to visible radiation. The array pixel size $8 \times 200 \mu \mathrm{m}$ provided the spectral resolution of the spectrograph indicated above. The total amplitude error of the detector was $\sim 10 \%$. Studies of similar CCD/luminophore detectors showed that the output signal amplitude linearly depended on the spectral radiation intensity. The output signal of the CCD array was fed to a PC. The device is described in more detail in [13].

We studied the influence of laser radiation on the plasma emission spectra by recording Al spectra for different laser pulse energies. Figure 2 shows plasma emission spectra in the range $30-120 \AA$ for the fixed

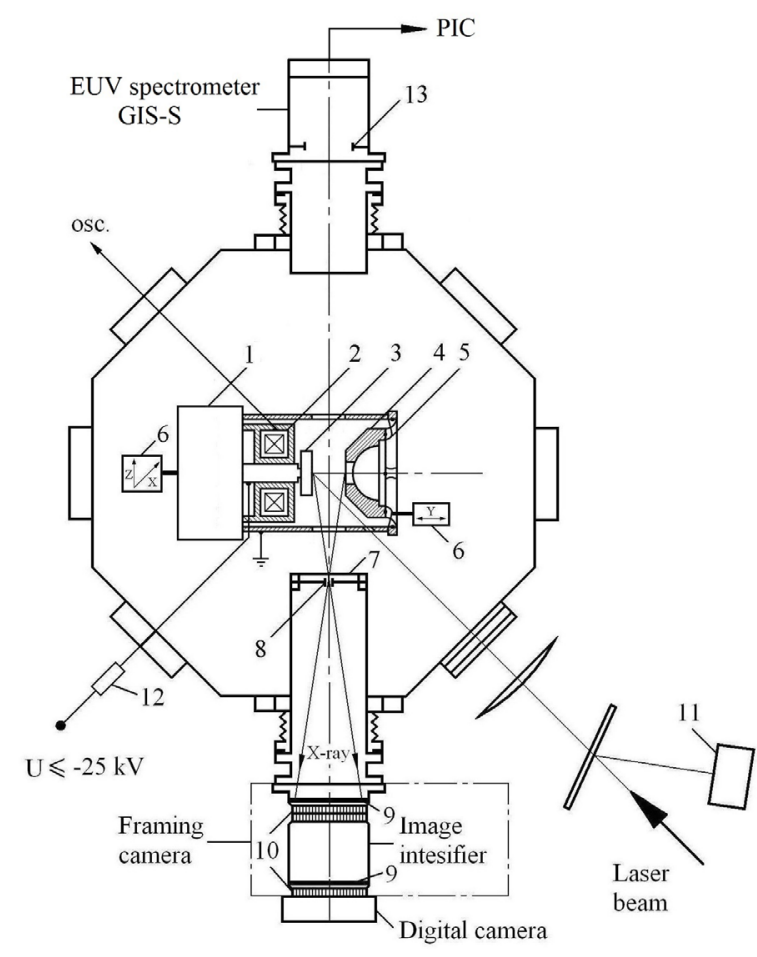

Fig.1. Experimental set-up. (1) capacitor; (2) Rogowski coil; (3) planar Al cathode; (4) anode; (5) flexible power input; (6) movable holders; (7) X-ray absorber; (8) pinholes; (9) MCP; (10) fiber optical interface; (11) calorimeter; (12) charging resistance of $100 \mathrm{M} \Omega$; (13) entrance slit of EUV spectrometer.

storage voltage $U=6 \mathrm{kV}$ (the discharge current amplitude is $I \approx 9 \mathrm{kA}$ ) and increasing laser pulse energy $J$. To determine the contribution of laser plasma emission into the discharge spectrum for each energy $J$, we also recorded the laser plasma emission spectrum in the absence of the discharge.

The obtained spectra showed that for $J<80 \mathrm{~mJ}$ the discharge plasma emission in this spectral range lies below the detection threshold. The laser plasma emission in the absence of the discharge can be detected for $J \geq 150 \mathrm{~mJ}$. The obtained spectra showed that the increase in $J$ from 80 to $110 \mathrm{~mJ}$ (Figs. 2a, b) resulted in the increase in the emission intensity and the shift of the $2 p^{4}-2 p^{3} 3 s$ transition line of Al VI to the $2 p^{2}-2 p 3 s$ line of Al IX, which suggest the increase in discharge plasma temperature $T_{e}$ and/or ion population. This effect is probably explained by the increase in the cathode material amount evaporated by laser radiation, which leads to the increase in the plasma compression degree in a micropinch and its heating. The increase in $J$ up to $150 \mathrm{~mJ}$ (Fig. 2c) results in the increase in the intensity of $2 p-3 d$ transition lines of the Al XI ion in the laser plasma and a drastic decrease in the mission lines of the discharge plasma. As $J$ is further increased, this tendency is preserved, so that for $J=250 \mathrm{~mJ}$ and the corresponding laser radiation density on the cathode $P=10^{12} \mathrm{~W} / \mathrm{cm}^{2}$ (Fig. 2d) and higher values of $J$, only the laser plasma emission is detected. This result shows that in the case of the high laser pulse energy and, therefore, the large mass of the plasma jet, the discharge current is not sufficient to obtain plasma pinching, in accordance with results $[9,14]$. 

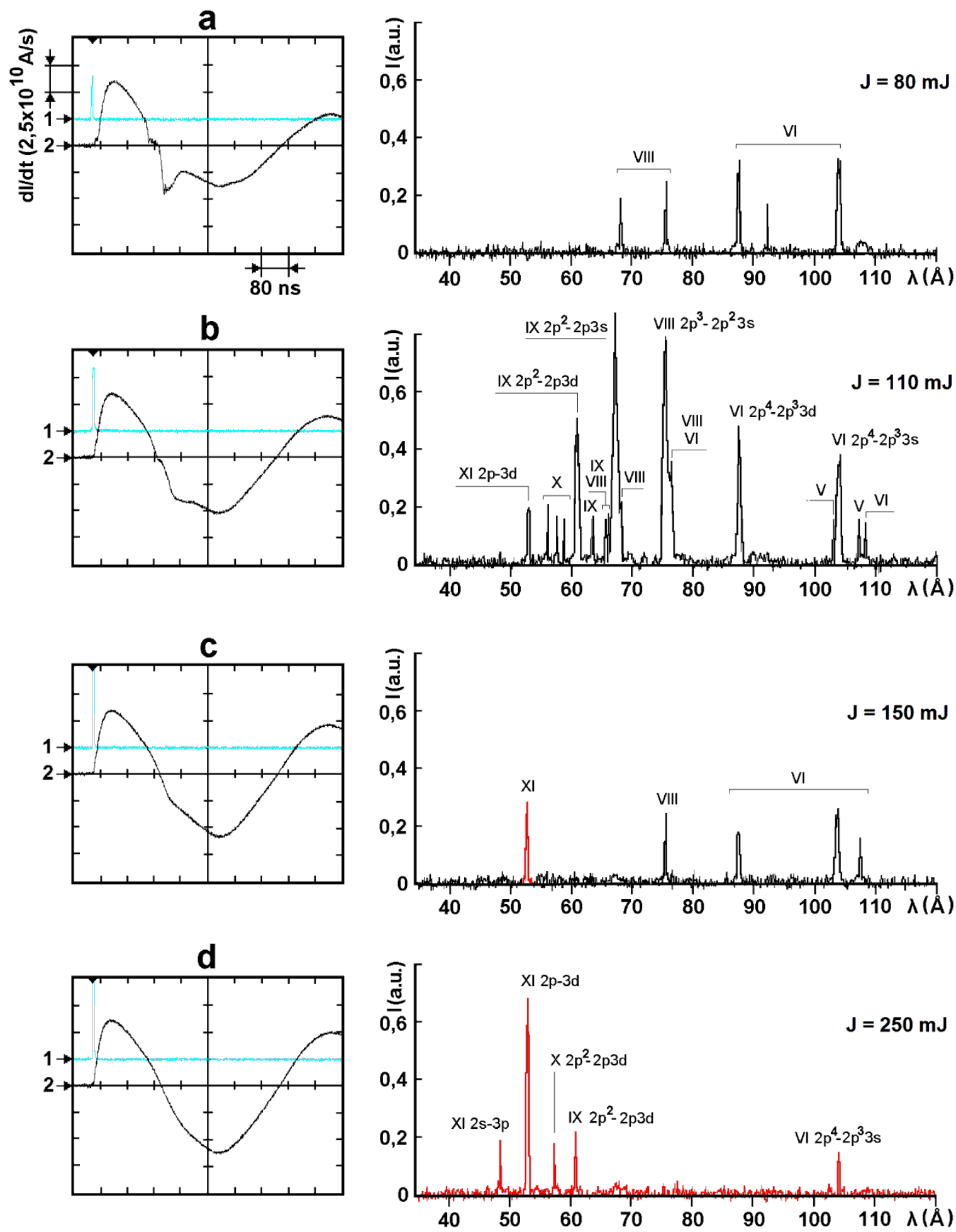

Fig. 2. Oscillograms of the discharge current derivative (at the left) and emission spectra of the Al plasma for $U=6 \mathrm{kV}$ and different $J$ (at the right). (1) laser pulse; (2) current derivative. The emission spectra of the discharge and laser plasma are shown by black and red curves, respectively. 
Figure 2 shows that the amplitude of the singularity in the oscillogram of the discharge current derivative (Fig. 2a, left column) characterizing the restriction on the current in a plasma channel due to the neck formation does not correlate with conditions of the maximum plasma heating (Fig. 2b, right column). It was shown in previous experiments that pinching predominantly occurs in the laser plasma produced during the discharge ignition [9]. For a low laser pulse energy and, therefore, a low linear density $N_{0}$ of the laser plasma in the discharge gap, the singularity amplitude achieves the maximum. The increase in $J$ leads to the "smoothing" of singularity. Thus, the initial value of $N_{0}$ plays a key role in the current flowing. For small $N_{0}$, the neck decay proceeds more efficiently, which can lead to the break of the current channel, the formation of a double dielectric layer and the anomalous acceleration of ions $[8,15]$. However, such a density proves to be insufficient for efficient plasma pinching and achieving the density and temperature values providing the detected radiation level.

The emission spectrum of the discharge plasma depends on the discharge current (Figs, 3a, b). One can see that, for the fixed laser pulse energy $70 \mathrm{~mJ}$, the increase in the voltage from 10 to $16 \mathrm{kV}$ corresponding to the increase in the current amplitude from 17 to $28 \mathrm{kA}$ results in the increase in the emission intensity by several times in the entire spectral range from 30 to $300 \AA$. The emission intensity predominantly increases (about 20 times) in the long-wavelength $230-270 \AA$ region of the quasi-continuum (unresolved emission arrays (UTA)). This suggests that the mean discharge plasma temperature decreases probably due to the increase in the plasma volume with a relatively low temperature produced during the development of the emission surface of the cathode with increasing the discharge current. The increase of $J$ from 70 to $200 \mathrm{~mJ}$ for $U=16 \mathrm{kV}$ leads to the further increase in the emission intensity and its redistribution to the shortwavelength region down to $100 \AA$. This indicates to the increase in the plasma temperature, which is also confirmed by the appearance of the Al XII $2 s-3 p$ lines of the He-like $\mathrm{Al}^{+11}$ ion (Fig. 3c). Our measurements showed that in this case the contribution of the laser plasma emission to the integrated spectrum does not exceed $5 \%$. The further increase in $J$ results in the decrease of temperature and emission intensity up to the complete cessation of plasma pinching. Note that emission spectra contain no traces of iron ions introduced to the total spectrum by the anode plasma emission, which means that the micropinch plasma temperature considerably exceeds the anode plasma temperature.

Unfortunately, because of the complex structure and specificity of VUV spectra of each element, at present there is no the universal procedure for determining the electron temperature $T_{e}$ and plasma density $N_{e}$ from them. These quantities are estimated using mathematical simulations. For some elements, in particular iron, a method is developed for determining the maximum temperature of electric discharge plasma by comparing its emission spectra with laser plasma spectra assuming the coronal equilibrium [10]. Experimental studies and mathematical simulations showed that the structure of VUV spectra of $\mathrm{Fe}$ ions in the laser plasma and the position of the maximum of the emission spectrum in the range $30-100 \AA$ are very sensitive to a change in the electron temperature. As $T_{e}$ increases, the ionization degree of $\mathrm{Fe}$ increases and the wavelength $\lambda_{\max }$ corresponding to the intensity maximum of the averaged spectrum for high-resolution spectra (or the intensity maximum for low-resolution spectrum) shifts to the blue. A comparison of these data obtained for laser plasma with data obtained for discharge emission spectra allows us to determine $T_{e}$ for the micropinch plasma. In this connection, we obtained the emission spectra of the discharge plasma with an iron cathode.

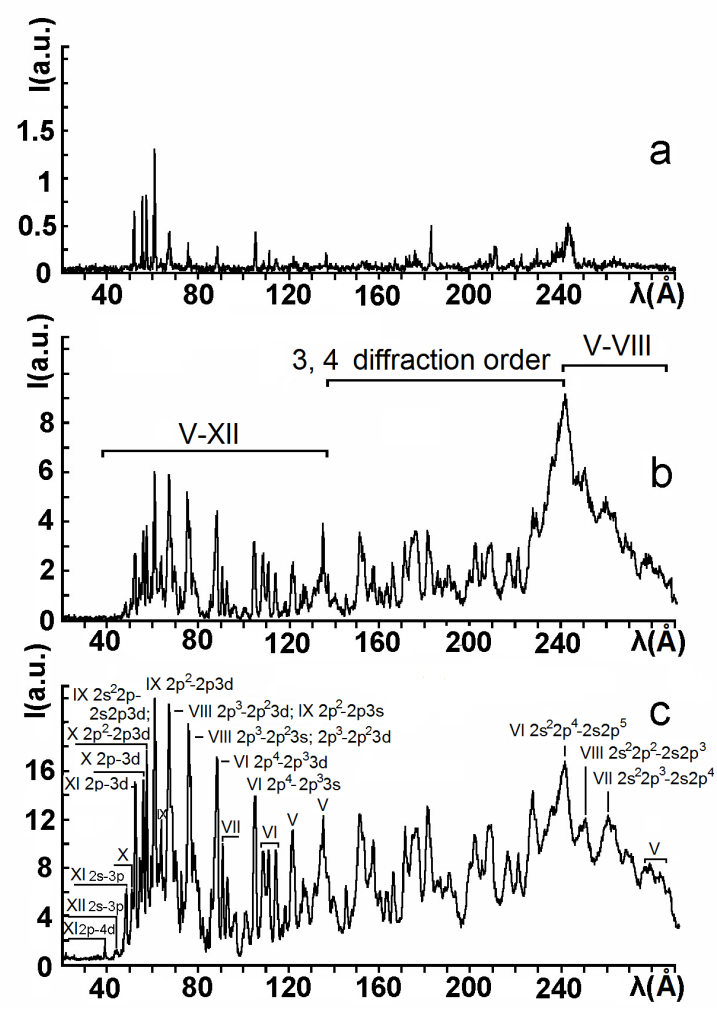

Fig. 3. Emission spectra of the Al discharge plasma for $U=10 \mathrm{kV}, I \approx 17 \mathrm{kA}, J=70 \mathrm{~mJ}$ (a); $16 \mathrm{kV}, 28 \mathrm{kA}, 70 \mathrm{~mJ}$ (b); and $16 \mathrm{kV}, 28 \mathrm{kA}, 200 \mathrm{~mJ}(\mathrm{c})$.

The behavior of emission spectra of $\mathrm{Fe}$ with changing the discharge current and laser pulse energy proved to be similar to that for $\mathrm{Al}$ emission spectra. The increase in the storage voltage (discharge current) for the laser pulse energy $80 \mathrm{~mJ}$ leads to the increase in plasma emission intensity (Figs. 4c, d). A detailed analysis of the structure of emission spectra in the short-wavelength almost did not change with increasing $U$ from 8 to $14 \mathrm{kV}$, which means that the temperature of the hot plasma region did not increase. The emission intensity considerably increased in the $220-260 \AA$ range, which means that the mean temperature of the emitting plasma volume decreased (this effect is more strongly manifested for $J<30 \mathrm{~mJ}$ ). The increase in $J$ up to $250 \mathrm{~mJ}$ (Figs. $4 \mathrm{a}-\mathrm{c}$ ) also leads to the increase in the 
emission intensity, which most strongly increases in the $30-150 \AA$ region, in particular, in the $66.3 \AA(3 \mathrm{~d}-4 \mathrm{f}$ transition of Fe XVI) and $67.2 \AA(3 d-4 p$ transition of Fe XVII) lines. However, any noticeably blue shift of the spectrum envelope maximum is absent, which means that the increase in $T_{e}$ is negligible [10]. The observed decrease in the ratio of the UTA emission intensity in the $160-200 \AA$ range to that in the short-wavelength region with increasing $I$ and $J$ corresponds to results [9] and is explained by the increase in the hot plasma volume.

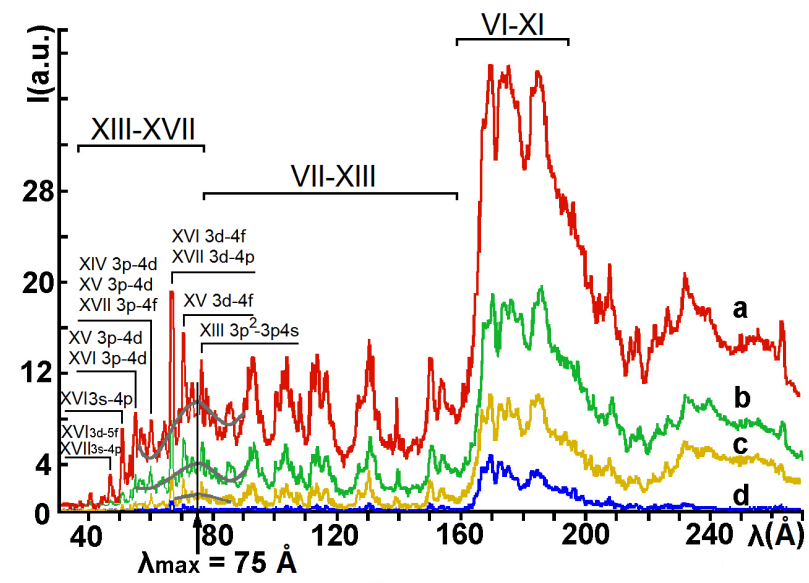

Fig. 4. Emission spectra of the $\mathrm{Fe}$ discharge plasma for $U=14 \mathrm{kV}, J=250 \mathrm{~mJ}$ (a); $14 \mathrm{kV}, 130 \mathrm{~mJ}$ (b); $14 \mathrm{kV}, 80 \mathrm{~mJ}$ (c); and $8 \mathrm{kV}, 80 \mathrm{~mJ}(\mathrm{~d})$. The arrow shows the approximate position $\lambda_{\max } \approx 75 \AA$ of the maximum of the averaged spectrum in the $30-90 \AA$ range.

Based on these data, we will estimate the maximum temperature of the discharge plasma by comparing the spectrum in the 30-90 $\AA$ region (Fig. 4a), where the overlap of diffraction orders is absent, with the VUV spectra of $\mathrm{Fe}$ ions in the laser plasma observed in experiments and synthesized in [10]. The comparison shows that the obtained spectrum is similar in its ion composition to the emission spectrum of the laser plasma. The analysis of the structure of the spectrum shows that the discharge plasma temperature lies in the range from 100 to $200 \mathrm{eV}$ (see Figs. $6-8$ in [10]). To estimate the temperature more accurately, we will use empirical formula (4) in [10]:

$\ln \left(\lambda_{\max }, A\right)=A \times\left(1 / T_{e}, \mathrm{keV}\right)+B$, where $A=0.082$ and $B=3.82$. The estimate of $T_{e}$ by this formula gives $T_{e} \approx 165 \mathrm{eV}$ for $\lambda_{\max } \approx 75 \AA$.

Thus, our studies have shown that:

(i) The SXR generation efficiency in a laser-triggered vacuum discharge depends on the relation between the laser pulse energy determining under these experimental conditions the mass of the evaporated plasma-producing cathode material, and the discharge current determining the plasma pinching rate. If the material mass is small, the amount of the produced plasma, its compression and heating degrees in a micropinch are insufficient for generating an intense SXR flow. On the contrary, in case of the mass excess, the current value is insufficient for the efficient plasma jet compression and heating. (ii) The intense UTA bands in the 230-270 $\AA$ and 160-200 $\AA$ ranges for $\mathrm{Al}$ and $\mathrm{Fe}$, respectively, are inherent in the emission spectra of the discharge plasma. They suggest the formation during pinching, along with the hot plasma nucleus with temperature $T_{e}>150 \mathrm{eV}$, also a relatively cold shell. There exists the range of laser pulse and discharge current parameters for which a considerable fraction of the radiation energy is concentrated in UTA.

(iii) The laser-induced vacuum discharge with the storage energy up to $30 \mathrm{~J}$ can be a promising for various applications as a SXR source with a broad line emission spectrum with parameters controlled by the laser pulse characteristics and the discharge current.

This work was supported by the Russian Foundation for Basic Research (grants nos. 15-02-03757, 16-02-01-140, 1702-00572).

\section{References}

1. C.R. Negus, N.J. Peacock, J. Phys. D: Appl.Phys., 12, 91 (1979)

2. A. Schulz, R. Burhenn, F.B. Rosmej, H.J. Kunze, J. Phys. D: Appl. Phys., 22, 659 (1989)

3. O.A. Bashutin, M.A. Alkhimova, E.D. Vovchenko, et al, Plasma Physics Reports, 39, 11, 900 (2013)

4. .Y. Vinokhodov, V.N. Krivtsun, A.A. Lash, et al, Quantrum Electron., 46, 1, 81 (2016)

5. D. Thanh-Hung, S. Yuhei, A. Goki, et al, Appl. Phys. Lett., 107, 12, 121101 (2015)

6. A. Sakdinawat, D. Attwood, Nature Photonics, 4, 840 (2010)

7. P.T. Springer, et al, J. of Quantitative Spectroscopy and Radiation Transfer, 58, 4-6, 927 (1997)

8. I.V. Romanov, V.L. Paperny, Yu.V. Korobkin, et al, Tech. Phys. Lett., 39, 388 (2013)

9. I.V. Romanov, V.L. Paperny, Yu.V. Korobkin, et al, Physics of Plasmas, 23, 023112 (2016)

10. A.P. Shevelko, D.E. Bliss, E.D. Kazakov, et al, Plasma Phys.Reports, 34, 11, 944 (2008)

11. V.V. Aleksandrov, V.A. Gasilov, E.V. Grabovskiy, et al, Plasma Phys.Reports, 40, 12, 939 (2014)

12. G.A. Vergunova, A.S. Grushin, A.A. Kologrivov, et al, Plasma Phys.Reports, 41, 5, 408 (2015)

13. A.P. Shevelko, O.F. Yakushev, Poverkhnost, 2, 51 (2003)

14. I.P. Tsygvintsev, A.Yu. Krykovskiy, V.A. Gasilov, et al, Mathematical Models and Computer Simulation, 8, 5, 595 (2016)

15. I.V. Romanov, A.A. Rupasov, A.S. Shikanov, et al, J. Phys. D: Appl. Phys., 43, 465202 (2010) 